\title{
Direct backside connection of optical fibers to GaAs detectors
}

Robert $W$. Ade and Eric R. Fossum

\author{
Center for Telecommunications Research \\ Columbia University \\ New York, New York 10027 \\ Michael A. Tischler
}

IBM Thomas J. Watson Research Center

Yorktown Heights, New York 10598

\begin{abstract}
$\underline{\text { ABSTRACT }}$
Photodetectors fabricated in GaAs have been vertically coupled to optical fibers using a cavity anisotropically etched in the backside of the GaAs substrate. The detector used was a Schottky photodiode amplified by an integrated MESFET. The cavity was etched from the backside using reactive ion etching (RIE). AlGaAs epitaxially grown on the substrate was used as an etch stop layer. A tapered optical fiber inserted in the backside cavity is accurately aligned to the detector and is mechanically stable. The vertical coupling approach is real-estate efficient and is particularly well suited for spatially parallel optical computing.
\end{abstract}

\section{INTRODUCTION}

Conventional metallic conductors carrying electronic signals between integrated circuits are susceptible to electromagnetic coupling which can result in parasitic capacitance, inductance, and noise pickup. Consequently, overall system performance suffers, especially at high bit rates. Signals transmitted photonically are immune to these effects and significant interest in the optical interconnection of electronic circuits has recently developed. A critical aspect of optical interconnection is the physical coupling between the optics and the integrated circuit. Fixed interconnection using optical fibers is likely to be the most reliable and practical technique.

Lateral fiber coupling ${ }^{1-3}$, while maintaining the planarity of the circuit topology, restricts interconnection to the periphery of the chip, thus limiting the number of interconnects. Vertical coupling, on the other hand, provides the smallest interconnect footprint (i.e., the fiber diameter) and is better suited for applications such as spatially parallel optical computing. However, vertical coupling of fibers has traditionally been plagued by mechanical instability and alignment difficulties.

We have previously demonstrated a technique to vertically couple optical fibers to photodetectors in silicon. 4 The interconnection was made by inserting a tapered single-mode fiber into a laser-etched cavity with a 20 um diameter. The interior of the cavity was doped to form a $p-n$ junction photodiode having a $45 \%$ external quantum efficiency and a modulation bandwidth of approximately $10 \mathrm{MHz}$.

A new fiber-optic interconnect structure, in which the detector is illuminated from the back side instead of the front, is presented in this paper. It is expected to show a fast response time and is designed to be compatible with high speed GaAs MESFET photoreceiver circuitry. An illustration of both coupling schemes appears in Fig. 1. Each uses an integrated, anisotropically etched cavity to position and anchor the fiber. The backside coupler makes greater demands on the etching technology used because of its larger size and the need to terminate etching within several microns of the front surface.

\section{BACKSIDE VERTICAL FIBER COUPLER IN GaAS}

The device structure for backside fiber coupling is shown in Fig. 2. The physical cross-section is similar to that used by Burrus and Dawson ${ }^{5}$ to couple optical fibers to surface emitting LED's, except that vertical cavity walls afforded by the anisotropic RIE process allow closer cavity spacing and better mechanical stability than the previously used wet etch. In the present work, an $\mathrm{Al} 0.35 \mathrm{Ga} 0.65^{\mathrm{As}}$ etch stop/window layer is employed, yielding a cavity with a flat bottom transparent to $830 \mathrm{~nm}$ light.

The detector structure chosen for this work was a Schottky photodiode with an integrated MESFET preamplifier, as shown in Fig. 3. The illuminated photodiode, when biased with a load resistor, modulates the MESFET gate voltage. The circuit, in principle, yields a reasonably large gain-bandwidth product. 


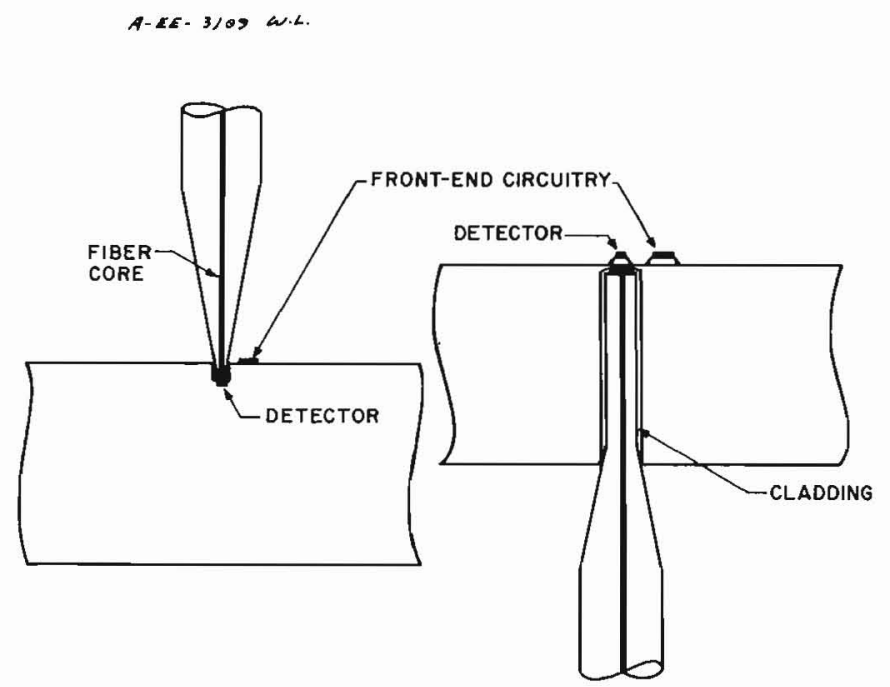

(a)

(b)

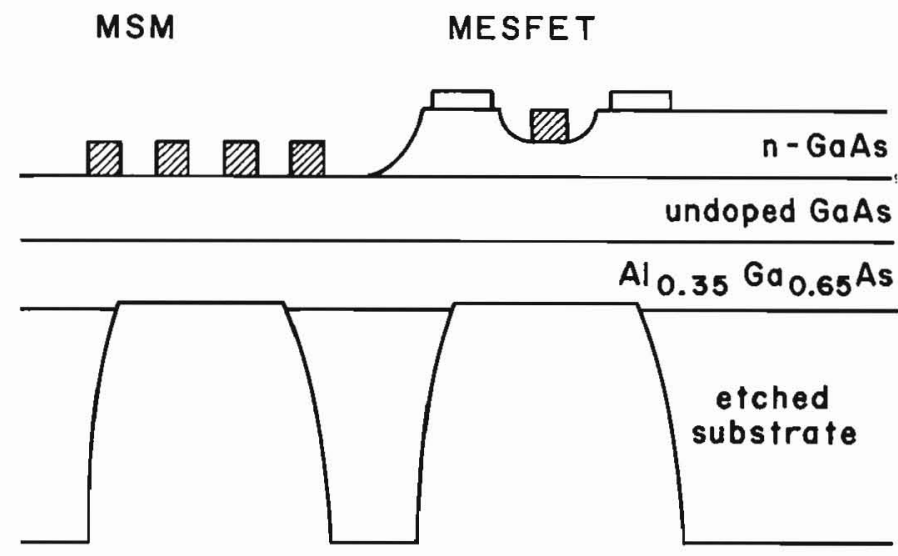

Fig. 1. Schematic diagram of

(a) frontside and (b) backside

fiber coupling with vertical cavities.

Fig. 2. Schematic cross-section of a backilluminated photoreceiver chip. The layer thicknesses are as follows: undoped AlGaAs, 4 um; undoped GaAs, 1 um; FET active layer $\left(\mathrm{N}_{\mathrm{D}}=5 \times 10^{16}\right), 0.4 \mathrm{um}$.

\section{Detector Fabrication:}

The epitaxial layers were grown in a barrel-type metal-organic vapor phase epitaxy (MOVPE) system using trimethylgallium, trimethylaluminum, arsine and disilane. The system was operated at 78 Torr and the growth temperature was $650^{\circ} \mathrm{C}$. A growth rate of

$0.07 \mathrm{um} / \mathrm{min}$ was maintained throughout the run. The substrates used were semi-insulating GaAs, oriented $(100), 2^{\circ}$ towards $\langle 011\rangle$. Aluminum composition was determined using low-temperature photoluminescence. An upper limit on the carrier concentration in the two undoped layers of $5 \times 10^{14} \mathrm{~cm}^{-3}$ was determined by $\mathrm{C}-\mathrm{V}$ profiling.

Mesas for FET isolation were defined using an etchant solution consisting of one part each $\mathrm{NH}_{4} \mathrm{OH}$ and $\mathrm{H}_{2} \mathrm{O}_{2}$ in 200 parts de-ionized $\mathrm{H}_{2} \mathrm{O}$. The mesas were overetched

$0.2 \mathrm{um}$ into the undoped GaAs layer. A thermaliy evaporated Au-Ge film 1500 A thick was patterned by liftoff to define ohmic contacts. These were annealed in a forming gas ambient for 40 seconds at $425^{\circ} \mathrm{C}$, using a rapid thermal processor. A $3000 \AA$ evaporation and liftoff of aluminum for the gate was the last step in the detector processing sequence. At that point, test chips were cleaved from the samples and characterized.
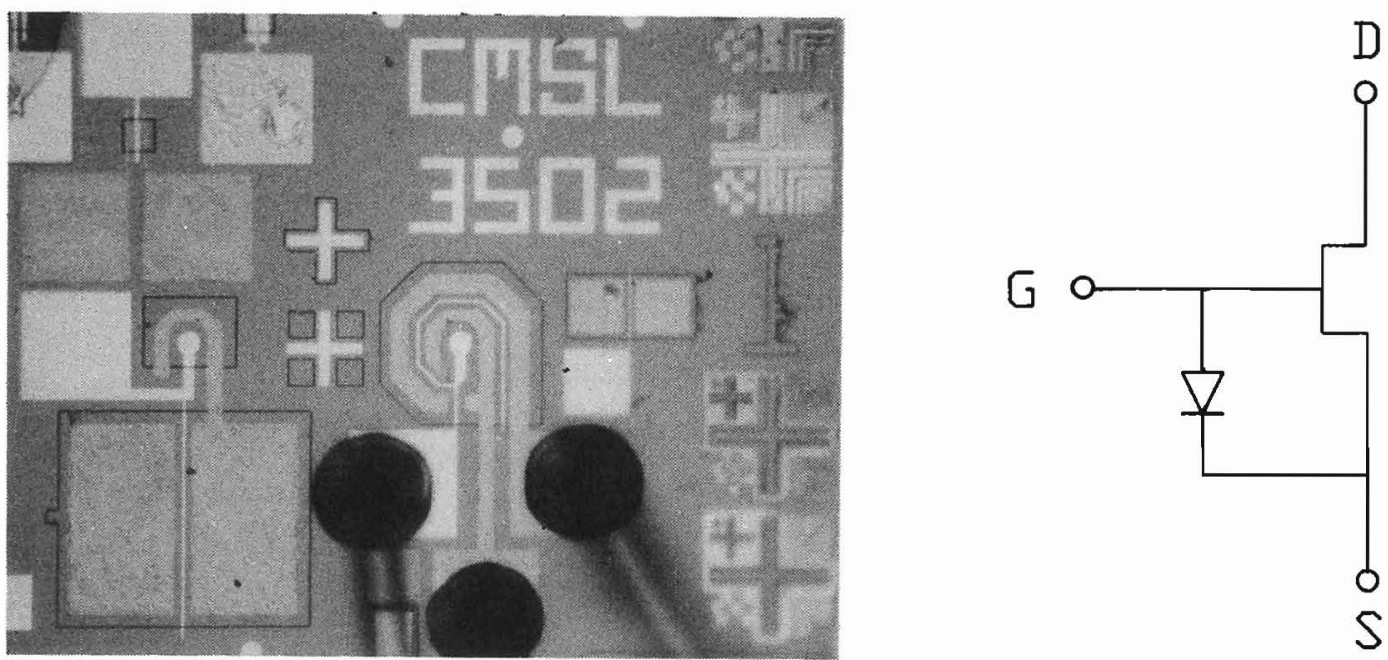

Fig. 3. (a) Photograph of GaAs detectors designed for fiber optic interconnection. The transistor on the right has gold wires attached to the bonding pads. (b) Circuit schematic of a diode-MESFET pair. 


\section{Cavity Formation:}

The device fabrication process is completed by a "customization" sequence which can be used to create fiber coupling sites for any fabricated device containing an appropriate etch stop layer. The devices are protected by mounting the sample face down on an oxidized silicon wafer with glycol phthalate. The GaAs substrate is thinned to approximately $200 \mathrm{um}$ and the backside is polished to allow photolithographic patterning of a Ni mask for the RIE. Front-to-back alignment is achieved using an IR camera attached to a Karl suss submicron aligner. This permits highly accurate positioning of the cavity with respect to the detector, as shown in Fig. 4.

The cavity is fabricated by a selective dry etch using $\mathrm{CCl}_{2} \mathrm{~F}_{2}$ as a source gas for chlorine and fluorine ions in an RF discharge. With this process it is possible to obtain a selectivity (etch rate ratio GaAs/AlGaAs) greater than 200.6 The low etch rate for AlgaAs allows it to be used as an etch stop layer, so that cavities reaching through several hundred um of GaAs can be terminated uniformly in the AlGaAs layer. Fig. 5 shows a cleaved cross-section of a typical etched cavity.
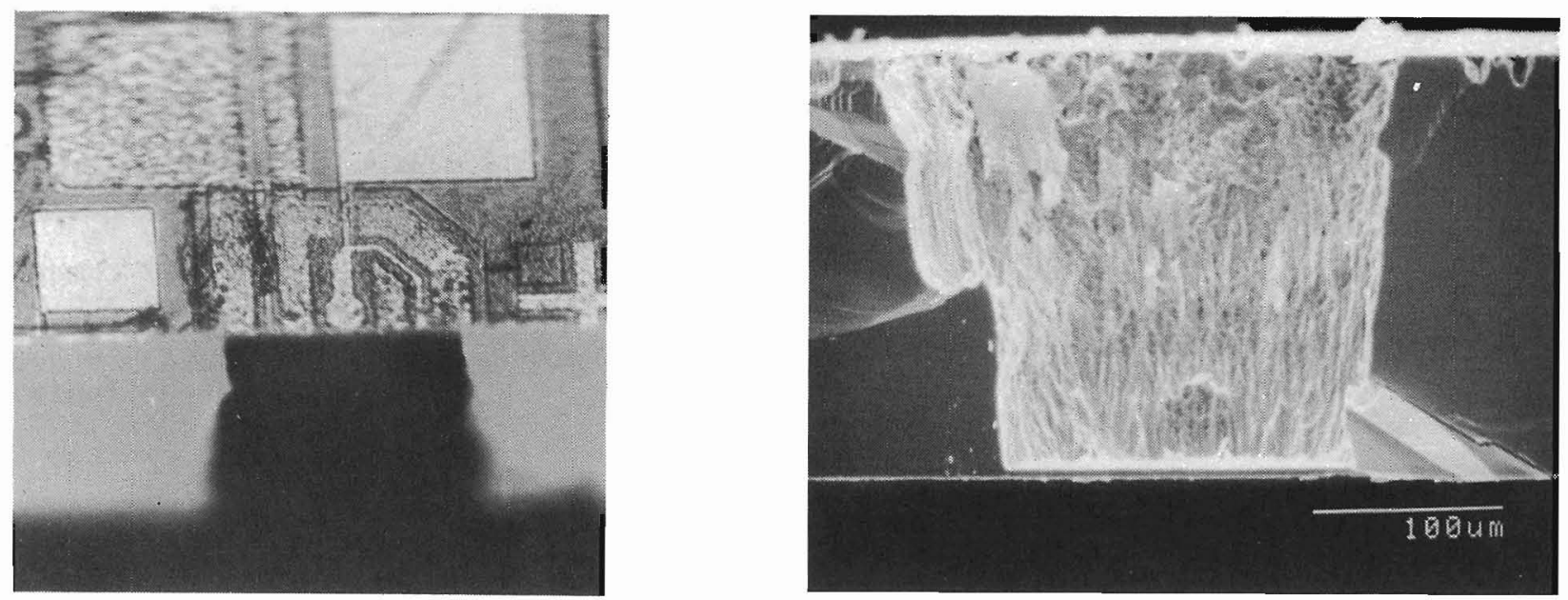

Fig. 4. Cut-away view of a cleaved sample demonstrating the alignment of a backside fiber-coupling cavity with a photodetector on the front surface.
Fig. 5. Cross-sectional scanning electron micrograph of an etched cavity terminating on an epitaxial AlgaAs layer (at bottom). The etch time was $125 \mathrm{~min}$.

The RIE chamber used has a conventional planar diode-type configuration. Typical process parameters are $5 \mathrm{~Pa}$ (38mTorr) pressure and an applied power density of $0.4 \mathrm{~W} / \mathrm{cm}^{2}$ at $13.56 \mathrm{MHz}$. The self-induced bias on the driven sample electrode is $-400 \mathrm{~V} D C$. Parameters used for cavity etching in the present work have given GaAs/AlGaAs ( $\mathrm{x}=0.35$ ) etch rate ratios between 30 and 50 . Etch rates between 2.0 and $2.5 \mathrm{um} / \mathrm{min}$. are obtained for GaAs etching in the vertical direction. There is also a lateral component $(10.2$ um/min.) which undercuts the $\mathrm{Ni}$ mask; this is difficult to avoid at pressures sufficient to give a high GaAs etch rate and a good selectivity. Lateral etching is undesirable since the cavity opening on the backside must remain smaller than the outer diameter of the coupled fiber. This keeps the thin epitaxial membrane from being destroyed by the fiber when it is inserted. A reduction in the diameter of the circular mask opening offsets the lateral etching problem, but also leads to a decrease in the vertical etch rate. The optimum process may be one in which the plasma parameters are varied during the etching. However, suitable cavities (including those shown above) can be fabricated with a constant parameter process.

\section{Fiber Preparation:}

The wet chemical etching process used for fiber tapering is similar to that described in Ref. 4. After the plastic sheath is mechanically stripped, the fiber is immersed in a stirred solution of buffered hydrofluoric acid. A smooth, tapered profile is obtained. The fiber tip is then scratched and cleaved to the proper length. $A$ photograph of a tapered fiber used in backside coupling appears in Fig. 6. 


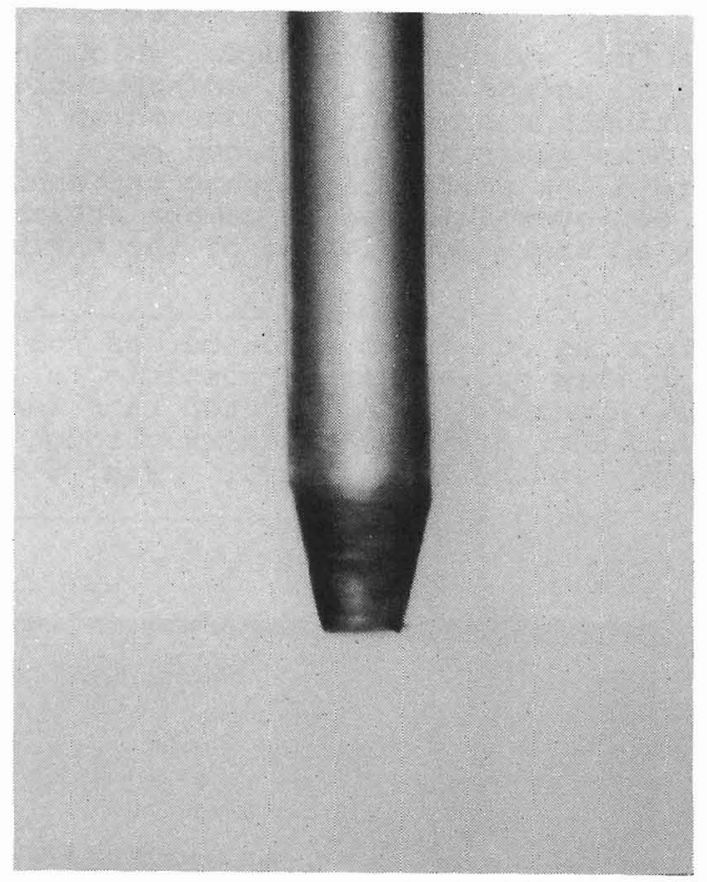

Fig. 6. Photograph of an optical fiber etched for $45 \mathrm{~min}$. in buffered $\mathrm{HF}$ at $40^{\circ} \mathrm{C}$. The diameter of the unetched portion of the fiber is 125 um.

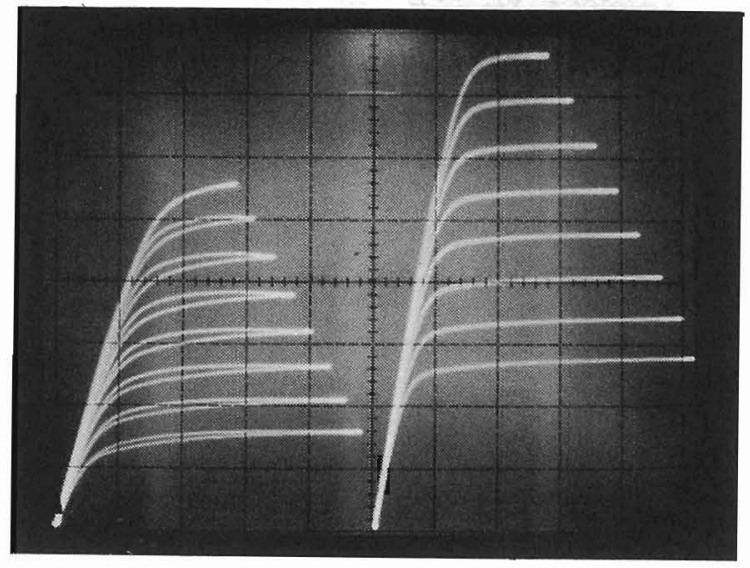

Fig. 7. Dark and illuminated transfer characteristics of a photodiodeMESFET pair. The horizontal and vertical scales are $2 \mathrm{~V}$ and $2 \mathrm{~mA}$ per division, respectively, and the gate voltage step is $0.2 \mathrm{~V}$. Incident optical power is $350 \mathrm{uW}$.

\section{DEVICE CHARACTERIZATION}

Electrical characteristics of the devices were measured prior to wafer thinning and backside cavity etching. The measured parameters for the pre-amp MESFET are in agreement with those calculated from the material properties and the device geometry. The pinchoff voltage is approximately 3.3 volts. The transconductance at zero gate bias is $25 \mathrm{~ms} / \mathrm{mm}$ with a gate-source capacitance of $2.4 \mathrm{pF}$. Based on the measured parameters, the transistor cut-off frequency is estimated to be $0.5 \mathrm{GHz}$.

Preliminary optoelectronic characterization of the detector has been performed using an $830 \mathrm{~nm}$ GaAs laser as the optical source. The probe beam (incident on the front surface) was approximately 40 um in diameter. Measurements were made to spatially profile the detector responsivity. These measurements indicate that in addition to the intended gate modulation mode of operation, a parasitic photoconductive mode is also present. This is probably due to the spreading of the optical signal throughout the mesa prior to absorption. Thus, although the gain-bandwidth product of the configuration is potentially large, very high speed operation may be prevented by relatively long carrier trap time constants. An example of the photoconductive mode is seen in Fig. 7, where the dark and illuminated photodiode-MESFET pair transfer characteristics are shown. The gate voltage was applied without a load transistor, thereby inhibiting optically-induced gate voltage modulation. However, significant photocurrent gain ( 10 $\mathrm{A} / \mathrm{W}$ responsivity) is still observed in this mode. The overall responsivity is reduced due to shadowing by the front surface metallization, which is eliminated when the detector is illuminated from the backside. Investigation of the performance of the structure under backside fiber illumination is currently in progress and will be reported separately.

\section{DISCUSSION}

The vertical coupling technique described in this paper is expected to have applications in several areas. While improved detector structures and front-end circuitry are being explored in this project for receiver applications, transmitter configurations are also of interest. Surface emitting LED and laser structures are being concurrently investigated, as well as multiple quantum well modulators. The key common component in these projects is the development of the backside vertical coupling cavity. It is believed that the RIE cavity formation process described in this paper will facilitate implementation of alternative optoelectronic device concepts. 


\section{ACKNOWLEDGEMENT}

The authors gratefully acknowledge useful discussions with R.E. Colbeth and D.V. Rossi of Columbia University. This work was supported by the NSF Center for Telecommunications Research.

\section{REFERENCES}

1. D.H. Hartman, M.K. Grace, and F.V. Richard, "An effective lateral fiber-optic electronic coupling technique suitable for VHSIC applications," J. Lightwave Technol. LT-4(1), 73-82 (1986).

2. P.R. Haugen, S. Rychnovsky, A. Husain, and L.D. Hutcheson, "Optical interconnects for high speed computing," Opt. Eng. 25(10), 1076-1085, (1986).

3. Y. Ota, R.C. Miller, S.R. Forrest, D.R. Kaplan, C.W. Seabury, R.B. Huntington, J.G. Johnson, and J.R. Potopowicz, "Twelve-channel individually addressable InGaAs/InP p-i-n photodiode and InGaAsP/InP LED arrays in a compact package," J. Lightwave Technol. LT-5(8), 1118-1222 (1987).

4. R.W. Ade, E.E. Harstead, A.H. Amirfazli, T. Cacouris, E.R. Fossum, P.R. Prucnal, and R.M. Osgood, Jr., "Silicon photodetector structure for direct coupling of optical fibers to integrated circuits," IEEE Trans. Electron Devices ED-34(6), 1283-1289 (1987).

5. C.A. Burrus and R.W. Dawson, "Small area high-current-density GaAs electroluminescent diodes and a method of operation for improved degradation characteristics," Appl. Phys. Lett., 17(3), 97-99 (1970).

6. K. Hikosaka, T. Mimura and K. Joshin, "Selective dry etching of AlGaAs-GaAs heterojunction," Jpn. J. Appl. Phys. 20(11), L847-L850, (1981). 